\title{
LA REFORMA DEL SISTEMA ELECTORAL ALEMÁN Y EL TRIBUNAL CONSTITUCIONAL FEDERAL
}

\author{
DIETER NOHLEN \\ Catedrático emérito de Ciencia Política \\ Universidad de Heidelberg
}

TRC, núm. 45, 2020, pp. 139-161

ISSN 1139-5583

\section{SUMARIO}

I. Consideraciones introductorias. II. El sistema electoral alemán en el debate clasificatorio: ¿mixto o proporcional? III. El sistema electoral alemán en sus reformas. IV. La injerencia del Tribunal Constitucional Federal en materia de reforma electoral. V. Conclusiones.

\section{CONSIDERACIONES INTRODUCTORIAS}

El sistema electoral alemán es percibido a nivel internacional —aunque no tanto en la misma Alemania - como modelo en el que pueda inspirarse una reforma electoral. Al mismo tiempo, existe un sorprendente desconocimiento sobre su funcionamiento y su clasificación, lo que genera confusión en los debates de reforma electoral que se refieren a él, dado que cada participante asume su propia definición del sistema alemán. Tampoco es bien conocido el debate suscitado al respecto en la misma Alemania y la reforma del sistema electoral que se produjo en 2013, muy determinada en sus dimensiones conceptuales por el Tribunal Constitucional Federal. Este análisis se propone abordar todas estas preguntas, la cuestión clasificatoria, la génesis y las reformas del sistema electoral alemán, el rol del Tribunal Constitucional Federal en ellas, especialmente el fundamento y alcance de su controvertida jurisdicción en esta materia electoral, dando énfasis en la reforma de 2013 y sus efectos no deseados que llevaron a la urgente necesidad de un rediseño del sistema.

Para una mejor comprensión del análisis politológico, ampliamos estas consideraciones introductorias a unas aclaraciones conceptuales, siguiendo a Max Weber en su afirmación de que, en las ciencias sociales, sin conceptos no se 
obtiene conocimiento ninguno y «no las conexiones 'de hecho' entre 'cosas' sino las conexiones conceptuales entre problemas están en la base de (su) labor»' ${ }^{1}$. Se puede precisar más esta idea para el caso concreto: entender los sistemas electorales en su relación material, presupone entender su orden conceptual. Entendemos por sistema electoral la forma de votar y la manera como se transforman en elecciones parlamentarias votos en escaños. En principio, son cuatro los elementos básicos de un sistema electoral: circunscripciones electorales, modos de candidatura, modos de votación, métodos de conversión de votos en escaños. Se puede añadir como interrogante, si existen o no barreras legales. El análisis de un sistema electoral dirigido a determinar los efectos sobre la representación política requiere mesurar la importancia relativa de cada uno de estos elementos. En conjunto, inciden en la clasificación de un sistema electoral, acorde con la distinción entre sistemas mayoritarios y sistemas proporcionales, y dentro de esta última categoría, entre diferentes tipos de sistemas proporcionales, por ejemplo entre representación proporcional personalizada (el caso alemán) y representación proporcional en circunscripciones plurinominales (el caso español) como resultado de concebir en el diseño electoral la representación proporcional como principio de representación o principio de decisión ${ }^{2}$. Los sistemas electorales tienen que cumplir con varias funciones. Como primer criterio de valoración de los sistemas proporcionales resulta común aplicar el grado de proporcionalidad que un sistema promueve o permite alcanzar entre votos y escaños de los partidos, al que vamos a dar por varias razones mucha atención en el análisis del sistema electoral alemán. Como segundo criterio figura el efecto de concentración de un sistema electoral, ejercido sobre la cantidad de partidos parlamentarios, el efecto sobre la estructura del sistema de partidos y la gobernabilidad que se logra en un sistema político. El tercer criterio consiste en el de (un mayor grado de) participación, en la mayor proximidad que podría sentirse entre votantes y elegidos, técnicamente promovida por poder elegir no solo entre partidos políticos, sino también entre personas, de poder entregar un voto personal. Se añaden a estos criterios básicos otros dos más: cuarto el criterio de la sencillez o transparencia del sistema, de modo que el votante pueda reconocer qué sucede con su voto, cómo se convierte en poder, y quinto el criterio de la legitimidad, dependiente del grado de excelencia técnica del sistema en relación a los otros criterios mencionados, y del grado de satisfacción de los electores con su desempeño, como resulte en debates sobre su reforma, en las que se manifiestan su crítica y su defensa en círculos académicos y en la opinión pública en general. Existe una conexión conceptual entre los criterios. A nivel empírico se manifiesta en una correlación, en su mayoría negativa,

1 Weber, M., Ensayos sobre metodología sociológica, 6a ed., Amorrortu, Buenos Aires. 2001, p. 57.

2 Nohlen, D., «Begriffliche Einführung in die Wahlsystematik» [Introducción conceptual en la sistemática electoral], en: Sternierger, D./ Vogel, B. (Eds.): Die Wabl der Parlamente und anderer Staatsorgane [La elección de los Parlamentos y otros órganos del Estado], de Gruyter, Berlín, 1969 y Sistemas electorales del mundo, CEC, Madrid 1981. 
entre las funciones por cumplir, que se expresa en la forma en que la maximización de una de ellas puede ocasionar la disminución en el cumplimiento de otra o todas las otras funciones. Finalmente, hay que advertir que no obstante la importancia de las reglas institucionales, constitucionales y legales en el efecto de los sistemas electorales, su desempeño empírico depende fundamentalmente del contexto histórico en sus múltiples expresiones, acorde con mi afirmación consistente en que el contexto hace la diferencia ${ }^{3}$.

\section{EL SISTEMA ELECTORAL ALEMÁN EN EL DEBATE CLASIFICATORIO: ¿MIXTO O PROPORCIONAL?}

No parece sencillo comprender el sistema electoral alemán. A veces tengo la impresión que hay tantos sistemas electorales alemanes como comentarios que a él se refieren. Es el resultado de enfocar conceptualmente este sistema, dado que combina elementos de diferente índole: combina el voto uninominal con el plurinominal, la circunscripción uninominal con la plurinominal, el principio mayoritario con el proporcional, cada uno con su específica función sistémica. Los especialistas alemanes abordan este sistema según la tradición proporcional del país como sistema plurinominal-proporcional, tomando como criterio clasificatorio el efecto que tiene el sistema sobre la composición de la Dieta Federal (el Bundestag): se impone la proporcionalidad entre votos y escaños. Es por eso que lo denominan «sistema proporcional personalizado». Esta designación en alemán significa: dentro del sistema proporcional se permite un voto uninominal-personal. Esto trae consigo que se aplica la regla decisoria mayoritaria para los candidatos que concurren en las circunscripciones uninominales, mecanismo para determinar una parte de los miembros de un grupo político en el parlamento en caso de que candidatos del respectivo partido político sean capaces de ganar escaños uninominales.

El mundo anglosajón, en general, aborda el sistema alemán a partir de su tradición mayoritaria y de su propio sistema electoral, que es uninominal-mayoritario. Los analistas anglosajones observan que en Alemania se añaden a las circunscripciones uninominales una igual cantidad de escaños plurinominales. En esto reside su idea que el sistema sea «mixto», al considerar la existencia de circunscripciones uninominales y plurinominales, incluso mitad-mitad ${ }^{4}$. Además, los especialistas anglosajones consideran que las circunscripciones pluripersonales tendrían la función de compensar las distorsiones originadas a nivel de las circunscripciones uninominales. Por eso, llaman el sistema electoral alemán a

3 Nohlen, D., El contexto hace la diferencia: reformas institucionales y el enfoque histórico-empírico. Ed. C. Zilla, UNAM, México, 2003.

4 RAE, D.W. The Political Consequences of Electoral Laws. Yale University Press, New Haven/London, 1967. 
menudo «additional member system» (los escaños proporcionales figuran como los añadidos) o «compensatory system» ${ }^{5}$. Recién, después de que Nueva Zelanda introdujo el sistema alemán, nombrándolo «mixed-member-proporcional» (MMP)-system (ver Royal Commission, 1986), en el mundo anglosajón se denomina al sistema alemán también según su copia: «mixed-member-proporcional»-system o (más corto) «mixed member»system. La óptica anglosajona no tendría mayor importancia si no albergara y divulgara un malentendido sobre el funcionamiento del sistema electoral alemán. Según esta corriente de análisis, el sistema electoral alemán es un sistema electoral mixto, clasificado como una tercera categoría, además de la representación por mayoría y proporcional. Está apreciado incluso como «the best of both worlds» ${ }^{6}$. En cuanto al impacto del sistema electoral alemán sobre el sistema de partidos, el que es importante por la asignación clasificatoria, es bien llamativo que hasta la década de 1970, la ciencia política no estaba segura de qué efectos ejercía. Maurice Duverger creyó, por ejemplo, que la concentración en el sistema de partidos en Alemania en los años sesenta y setenta se debía al elemento mayoritario del sistema electoral consistente en la elección de personas en las circunscripciones uninominales ${ }^{7}$.Vale insistir, sin embargo, que el sistema de representación proporcional personalizada es proporcional, y la jurisdiccional constitucional al respecto, poco tomada en cuenta en los análisis clasificatorios del sistema, lo confirma, como vamos a ver más adelante.

\section{EL SISTEMA ELECTORAL ALEMÁN EN SUS REFORMAS}

A continuación, analizaremos la evolución del sistema electoral alemán en su funcionamiento práctico. Han sido varias las reformas que se han llevado a cabo y que llegaron a cambiar incluso el tipo de representación proporcional. El tipo al que el mundo científico y político iba a referirse como «el sistema alemán», se

5 Ver, por ejemplo, Irvine, W.P., 'Additional-Member' Electoral Systems, en: Lijphart, A. / GrofMAN, B. (Eds.): Choosing an Electoral System: Issues and Alternatives, Nueva York: Praeger, Nueva York, 1984; Rose, R., International Encyclopedia of Elections. Congressional Quarterly, Washington, D.C. 2000; Shugart, M.S., Wattenberg, M., Mixed-Member Electoral Systems: The Best of Both Worlds? Oxford University Press. Oxford, 2001, Gallagher, M., Mitchell, P., (Eds.), The Politics of Electoral Systems. $2^{\mathrm{a}}$ ed., Oxford University Press, Oxford, 2008.

6 Shugart, M.S., Wattenberg, M., Mixed-Member Electoral Systems: The Best of Both Worlds? Oxford University Press. Oxford, 2001.

Ver, entre otros, Lijphart, A., Advances in the Comparative Study of Electoral Systems, en World Politics 36, 1984; Shugart, M.S., Wattenberg, M., Mixed-Member Electoral Systems: The Best of Both Worlds? Oxford University Press. Oxford, 2001.Jesse, E., Verhältniswahl und Gerechtigkeit [Representación proporcional y Justicia], en Strohmeier, G. (Ed.): Wahlsystemreform. Sonderheft der Zeitschrift für Politikwissenschaft [Reforma al sistema electoral. Cuaderno especial de la Revista de Ciencia Política], Nomos, Baden-Baden, 2009, p. 117.

7 Duverger, M., «Which is the Best Electoral System?», en, Lijphart, A. / Grofman, B. (Eds.): Choosing an Electoral System. Issues and Alternatives. Praeger, Nueva York, 1984, p. 37. 
estableció solo después de unas reformas iniciales en los años cincuenta. Desde allí, sus características básicas quedaron inalteradas hasta 2013.

\section{Génesis y comprensión del sistema electoral alemán}

En el Parlamentarischer Rat (Consejo Parlamentario que era constituyente) «a todos los partidos (era común) la determinación de no repetir los errores del sistema de Weimar» ${ }^{8}$. La lección de Weimar consistió en primer lugar en el propósito de no incluir el sistema electoral en la Constitución. Debía ser legislado y reformable por simple ley. En segundo lugar, nadie quería volver a la representación proporcional pura de aquella República. Las opciones del sistema electoral de los partidos políticos eran ciertamente muy contrarias. El partido socialdemócrata (SPD) quería introducir, junto con los pequeños partidos, un sistema de representación proporcional; la democracia cristiana (CDU / CSU) el sistema de mayoría relativa en circunscripciones uninominales. Finalmente se impuso en los largos debates, en las que intervinieron también las potencias ocupantes ${ }^{9}$, el SPD con la ayuda de los partidos pequeños, especialmente los Liberales del FDP, respecto al principio de representación. Si bien la Constitución (Grundgesetz / Ley Fundamental) fue aprobada por unanimidad, en el caso del sistema electoral no se consiguió alcanzar el consenso de todos los partidos. Sin embargo, se creyó haber encontrado un compromiso entre un sistema electoral uninominal (Personenwabl) y un sistema electoral de listas (Listenwabl), trasladando la controversia clásica entre representación por mayoría y representación proporcional al nivel de los elementos técnicos. De verdad, a ese nivel, se combinaron en el nuevo sistema electoral circunscripciones uninominales con plurinominales, la regla decisoria de mayoría relativa con la de proporcionalidad, lo que correctamente percibió James K. Pollock en uno de los primeros análisis del «nuevo sistema combinado alemán» de tendencia proporcional, reuniendo los «advantages of a straight P.R. system, inaccurately representing all of the main streams of opinion»..., "a new synthesis of antithetical electoral systems which... meets any fair set of criteria for a good representative system»... (y) promises to emerge as a real contribution to representative government» ${ }^{10}$.

8 WILD, M., Die Gleichbeit der Wabl. Dogmengeschichtliche und systematische Darstellung [La igualdad de la elección. Exposición dogmática histórica y sistemática], Duncker \& Humblot, Berlín, 2003, p. 81.

9 Ver LANGE, E.H.M., Wablrecht und Innenpolitik [Derecho electoral y política interior], HAIN-VERLAG, Meisenheim am GLAN, 1975; Jesse, E., Wablrecht zwischen Kontinuität und Reform. Eine Analyse der Wablsystemdiskussion und der Wablrechtsänderungen in der Bundesrepublik Deutschland 1949-1983 [El sistema electoral electoral entre continuidad y reforma. Un análisis de la discusión sobre el sistema electoral y sus cambios en la República Federal de Alemania 1949-1983], Droste, Düsseldorf, 1985; WILD, M., Die Gleichbeit der Wabl. Dogmengeschichtliche und systematische Darstellung [La igualdad de la elección. Exposición dogmática histórica y sistemática], Duncker \& Humblot, Berlín, 2003.

10 Pollock , J.K. «The Electoral System of the Federal Republic of Germany - A Study in Representative Government», en, American Political Science Review 46, 1952: pp. 1061, 1066 y 1067, respectivamente. 
La ley electoral preveía, para la elección del primer Bundestag en 1949, una proporción de $60 \%$ de los representantes elegidos individualmente en circunscripciones uninominales y con mayoría relativa y de $40 \%$ de representantes elegidos por listas en circunscripciones plurinominales, formadas en los Estados federados (Länder), con adjudicación proporcional de los escaños. Para participar en la distribución de los escaños se aplicaba la barrera legal del 5\% a nivel de los Länder. Todos estos elementos incluso podrían dar a suponer una preponderancia de lo mayoritario en el sistema. Precisamente no se sabía cómo clasificar el nuevo sistema electoral, ni evaluarlo en términos de su posible impacto. Hay que recordar que esta combinación de diferentes elementos de sistemas electorales se llamaba sistema de mayoría proporcionalizado en el momento de su invención al final de la República de Weimar. Se pensó que el sistema pertenecía a la categoría de los sistemas mayoritarios ${ }^{11}$. El comentario a la Ley Federal Electoral definía aún en la década de los años 1960 la representación proporcional personalizada como «sistema electoral de conexión que tiene como fundamento el sistema electoral de mayoría (sic), superpuesto luego por la representación proporcional» ${ }^{12}$. Sin embargo, ya en 1949, el fundamento del sistema alemán estaba en la representación proporcional. En términos de los tipos de sistemas electorales se optó en 1949 por un sistema de representación proporcional en circunscripciones plurinominales con barrera legal de alcance regional.

\section{El sistema electoral anterior a la reforma de 2013}

En las elecciones parlamentarias de 1957, Alemania votó por primera vez según el sistema electoral que iba a denominarse «sistema alemán» y figurar más bien fuera de Alemania por decenios como sistema electoral modelo. Antes, en 1953 se había igualado la cantidad de escaños distribuidos en circunscripciones uninominales y plurinominales, e introducido el sistema de dos votos, el primero por candidatos (Personenwabl) y el segundo por las listas de los partidos a nivel de los Länder (Listenwahl). Luego, en 1956, se trasladó el nivel de aplicación de la barrera legal del cinco por ciento de los Länder a la federación. Con estas reformas se completó la representación proporcional personalizada. Posteriores reformas no impactaron en el tipo de sistema proporcional, por ejemplo, en 1985, la sustitución del método d'Hondt por el método Hare/Niemeyer, y este, en 2008, por el método Sainte Laguë/Schepers. Tampoco impactó en él la unificación de

11 Al igual que en Italia, en 1993, cuando se pensaba haber adoptado, con la creación de muchas circunscripciones uninominales en un sistema de representación proporcional, un sistema mayoritario (Así D'alimonte, R., «Italy: A Case of Fragmented Bipolarism», en: Gallagher, M. / Mitchell, P. (Eds.): The Politics of Electoral Systems, $2^{\text {a }}$ ed., Oxford University Press, Oxford, 2008, p. 256.

12 Seifert, K.H., Das Bundeswablgesetz [La Ley Federal Electoral], $2^{\mathrm{a}}$ ed. Berlín - Francfort del Meno, 1965 , p. 20. 
Alemania. Esto implicó solo un aumentó la cantidad de miembros del Bundestag, hasta que se logró reducir su cantidad a principios de nuestro siglo. La reciente reforma de 2013 cambió elementos claves del sistema, con importantes consecuencias en su funcionamiento y su valoración, de modo que se impone diferenciar estrictamente entre el sistema electoral alemán anterior a la reforma de otro posterior a ella.

A partir de 2002 el Bundestag contaba, en principio, con 598 miembros. El número puede aumentar debido al fenómeno de los mandatos excedentes (Überhangmandate) que nos va ocupar más tarde. El país se dividió, y sigue dividido, en 299 circunscripciones uninominales y en 16 circunscripciones plurinominales que corresponden a los Länder. Cada elector dispone de dos votos: el primer voto (Erststimme) para la elección directa de un diputado en las circunscripciones uninominales por mayoría relativa; el segundo voto (Zweitstimme) para votar por una lista cerrada y bloqueada de partido a escala de los Länder. Este segundo voto determina cuántos diputados envía cada partido al Bundestag. Para este cálculo de distribución proporcional, hasta la reforma de 2013 se sumaban los segundos votos que los partidos han conseguido a nivel de las circunscripciones plurinominales, a escala nacional. Para atribuir los 598 escaños a los partidos se tomaba en cuenta sólo a aquellos (con la excepción de los partidos de minorías nacionales), que habían obtenido al menos el cinco por ciento de los votos a nivel nacional o tres escaños en unas circunscripciones uninominales. Esta distribución, o sea la composición partidista del Bundestag, era determinada mediante una doble utilización de la fórmula de conversión de votos en escaños, el método Sainte Laguë/ Schepers. La primera operación se refería a la distribución de los escaños a los partidos a nivel nacional. Por medio de la segunda operación se repartían los escaños ya asignados a cada partido a nivel nacional entre sus distintas listas plurinominales a nivel de los Länder. Una vez determinado el número de escaños que correspondían a cada partido en cada Land, se sustraían los escaños obtenidos en las circunscripciones uninominales (Direktmandate) en cada Land, es decir, éstos se restaban del número de escaños adjudicados a las listas de partidos plurinominales. Si el número de escaños conseguido por un partido en las circunscripciones uninominales con los primeros votos sobrepasaba la cantidad que correspondía a las listas plurinominales por los segundos votos, los partidos conservaban estos escaños excedentes, aumentándose transitoriamente el número total de diputados en el Bundestag.

La descripción del sistema electoral alemán antes de la reforma de 2013 confirma la tesis clasificatoria que es proporcional en su mecanismo institucional. La composición del Bundestag no puede resultar no-proporcional, excepto en caso de alta cantidad de escaños excedentes, y en la práctica, la relación entre votos y escaños resultó siempre muy proporcional, con sus propios matices en cada elección debido a factores de contexto: el sistema de partidos concurrentes y el comportamiento electoral del electorado. Esta proporcionalidad se estableció en el inicio mismo de calcular y conformar el resultado electoral en base a los votos 
entregados. La proporcionalidad no resultó ser algo añadido, tampoco resultó ser compensatoria. Excluyó a nivel de los principios de representación su clasificación como mixto. Lo que sí se combina son elementos que se perciben como antitéticos (circunscripciones uninominales versus plurinominales, voto uninominal versus lista cerrada y bloqueada, principio mayoritario versus proporcional), todos y cada uno con funciones específicas en una estructura básica de sistema de representación proporcional.

\section{Efectos del sistema electoral antes de su reforma de 2013}

En un principio eran muchos los mitos existentes sobre los efectos del sistema alemán, muy dependiente de su percepción clasificatoria. Los defensores del sistema de mayoría relativa supusieron que el sistema proporcional produciría multipartidismo e ingobernabilidad ${ }^{13}$, haciendo revivir en alguna forma la experiencia de Weimar. Cuando se produjo el efecto contrario, una concentración hacia un sistema de dos partidos y medio (el Zweieinhalbparteiensystem de los partidos CDU/CSU, SPD y FDP), esta corriente de pensamiento trató de aprovechar la situación histórica para introducir un sistema electoral de tendencia mayoritaria (mehrbeitsbildendes $W$ ablsystem), intento que falló, cuando los socialdemócratas se retiraron del proyecto legislativo. En la medida que se fortaleció la estabilidad del sistema de partidos, los protagonistas de una reforma mayoritaria iban a silenciarse y conformarse con el sistema electoral vigente, mientras que la corriente de pensamiento opuesta, muy vinculada con la clasificación del sistema proporcional personalizado como mixto, iba a considerar el sistema electoral gracias a sus elementos mayoritarios como causante de tal concentración. Esta tesis, muy cuestionable por sí misma debido a la sobrevaloración de lo institucional en contextos multifactoriales, fue descartada al cambiarse el sistema de partidos a partir de finales de los ochenta con el auge de los Verdes y la reunificación de Alemania que aportaba otro partido más, la posterior Izquierda (Die Linke). Además de la fragmentación del sistema de partidos, ella misma hizo que el sistema electoral mostrara algunos efectos que condujeron a su crítica. En el centro del creciente malestar entró el fenómeno de los escaños excedentes que aumentaron en cantidad y distribución desigual debido al descenso en votos primero del SPD, el que no pudo seguir participando igual en estos escaños, y luego de la CDU, por lo que se aumentó el número de escaños excedentes hasta cuestionar la legitimidad del sistema. En términos científicos, se daba a entender que la relación causal entre sistema electoral y sistema de partidos es bidireccional o sistémica, y que en esta

13 Ver Sternberger, D., Die große Wablreform. Zeugnisse einer Bemühung [La gran reforma electoral. Testimonios de un esfuerzo], Westdeutscher Verlag, Opladen, 1964; Hermens, F.A., Demokratie oder Anarchie [Democracia o anarquía], Westdeutscher Verlag, Opladen (primero en inglés en 1941), 1968. 
relación cambian las funciones de emisor y receptor entre los factores. En términos políticos, cuando cambia la estructura de efectos según los actores, o sea, cuando unos se ven estructuralmente perjudicados, esto origina sin lugar a duda debates de reforma. En las elecciones de 2009, de los en total 24 escaños excedentes la CDU recibió 21, su partido hermano, la CSU de Baviera, tres. La socialdemocracia impulsaba la compensación proporcional de los escaños excedentes, involucrando el Tribunal Constitucional Federal en búsqueda de lograr esta reforma. El cambio en el sistema de partidos antecedía la reforma electoral.

\section{Hacia la reforma del sistema proporcional}

Respecto a las razones de esta reforma, hay que distinguir entre una razón inicial y razones estructurales que se sumaron en el proceso mismo de la reforma. La razón inicial consistía en el efecto no intencionado del sistema, el valor de logro inverso, posible producto del cálculo de repartición de los escaños relacionado con los escaños excedentes no compensados. Consiste en que un aumento en segundos votos de lista hubiera podido llevar a una pérdida en escaños de la respectiva lista de partido del Estado miembro, o a que una pérdida en votos de lista hubiera ocasionado un aumento en los escaños de un partido ${ }^{14}$. El Tribunal Constitucional Federal sentenció el 3 de julio de 2008 que este efecto era inconstitucional y obligó al legislador a establecer una regulación compatible con la Constitución ${ }^{15}$. Esta necesaria reforma abrió un debate electoral general que incluía otras posibles reformas, incluso alternativas al tipo de sistema de representación proporcional personalizada, pero principalmente dirigidas a eliminar la desproporcionalidad originada por la no-compensación de los escaños excedentes ${ }^{16}$. Una primera reforma de diciembre 2011, propuesta por el gobierno de coalición de la Democracia Cristiana

14 Meyer, H., «Der Überhang und anderes Unterhaltsame aus Anlass der Bundestagswahl 1994» [Los escaños excedentes y otras cosas divertidas en ocasión de las elecciones al Bundestag de 1994], en: Kritische Vierteljabresschrift für Gesetzgebung und Rechtswissenschaft 77 (4), 1994.

15 Véase Nohlen, D., «La reforma necesaria al sistema electoral alemán: el principio de igualdad del valor de logro del voto como idée fixe en la sentencia del Tribunal Constitucional Federal del 3 de julio de 2008», en: Justicia Electoral, Tribunal Electoral del Poder Judicial de la Federación, $3^{\mathrm{a}}$ Época, vol. 1, nº, México, 2009.

En su sentencia del 3 de julio de 2008 (registrada en BverfG, 2 BvC 1/07 y 2 BvC 7/07) se dijo: «(El peso negativo del voto, consistente en que) un aumento de votos del partido puede causar una pérdida de escaños en las listas nacionales o una pérdida de segundos votos un aumento en los asientos de las listas regionales,» viola el artículo 38 párrafo 1 de la Ley Fundamental, según el cual el Bundestag alemán tiene que surgir de elecciones universales, igualitarias, directas y secretas. Se ordenó que el legislador, a «no más tardar del 30 de junio de 2011, llevara a cabo una reglamentación conforme al precepto constitucional» (véase Nohlen, 2009).

16 Véanse Strohmeier, G., Wablsystemreform. Sonderheft der Zeitschrift für Politikwissenschaft [Reforma del sistema electoral. Cuaderno especial de la Revista de Ciencia Política], Nomos, Baden-Baden, 2013; PoIER, K., Wahlsysteme im internationalen Vergleich — ein Überblick [Sistemas electorales en comparación internacional — Una visión panorámica], en: PoIER, K. (Ed.): Demokratie im Umbruch. Perspektiven einer 
y los Liberales, que se restringió a reducir el efecto no-deseado del voto de logro negativo, fue también declarada inconstitucional el 25 de julio de 2012, entre otras razones por no limitar suficientemente a los escaños excedentes, cuyo número era previsible de aumentar debido a la mayor fragmentación del sistema de partidos. En la sentencia se objetó que los escaños excedentes podrían ocurrir en tal cantidad que podrían «cancelar el carácter fundamental de representación proporcional de la elección del Bundestag». Otra razón novedosa y de gran calibre consistía en que la reforma no había incluido a la oposición política en la toma de decisión al respecto. Esta condición política, implantada por el Tribunal Constitucional Federal - aunque el sistema electoral alemán en su origen tampoco había sido acordado por consenso- iba a favorecer la compensación de los escaños excedentes como elemento clave de la reforma electoral por legislar, lo que era un postulado de la Socialdemocracia y de Los Verdes.

\section{El sistema electoral alemán reformado}

La reforma del sistema electoral de mayo de $2013^{17}$ dejó algunos elementos claves del sistema invariables ${ }^{18}$. Sigue siendo un sistema combinado de representación proporcional, pero ahora de tipo compensatorio. No cambió el número de miembros del Bundestag (en teoría 598). El país sigue dividido en 299 circunscripciones uninominales y en 16 circunscripciones plurinominales. Los electores continúan disponiendo de dos votos: el primero para los candidatos en las circunscripciones uninominales por mayoría relativa; el segundo voto para votar por una lista cerrada y bloqueada de partido a escala de los Länder. Este segundo voto sigue determinando cuántos diputados envía cada partido al Bundestag. Sin embargo, se ha fortalecido aún más el carácter proporcional del sistema de manera que conviene clasificarlo ahora como compensatorio, buscando en cuanto a objetivos el más alto grado de proporcionalidad entre votos y escaños en detrimento de otras funciones del sistema electoral.

El nuevo sistema electoral aspira a la proporcionalidad en dos niveles de adjudicación de escaños, en cada uno con diferente punto de referencia ${ }^{19}$. Primero, la proporcionalidad se propone a nivel de los Länder, dado que estos constituyen ahora

Wablrechtsreform [Democracia en transformación. Perspectivas de una reforma al derecho electoral], Böhlau, Vienna-Colonia-Graz, 2009.

17 Bundesgsetzblatt BGBl I, 2013: 1082 y sig.

18 Ver Dehmel, N., Jesse, E., «Das neue Wahlgesetz zur Bundestagswahl 2013. Eine Reform der Reform der Reform ist unvermeidlich» [La nueva ley electoral para la elección del Bundestag de 2013. Una reforma de la reforma de la reforma es imprescindible], en: Zeitschrift für Parlamentsfragen 44, 2013.

19 Ver Grotz, F., «Der Mandatsausgleich im neuen Bundestagswahlsystem», en: Oppelland, TH. (Ed.): Das deutsche Wablrecht im Spannungsfeld von demokratischer Legitimität und politischer Funktionalität [El sistema electoral alemán en tensión entre legitimidad democrática y funcionalidad política], Berliner Wissenschaftsverlag, Berlín, 2015. 
verdaderas circunscripciones electorales plurinominales, a las que se asignan los 598 escaños (mitad uninominales, mitad plurinominales) según el criterio de la población, y luego distribuyen los escaños por partido en una primera operación de distribución proporcional. Se suprimió entonces la conexión de las listas de partidos entre diferentes Länder que era la base inicial del sistema alemán en la distribución proporcional de los escaños a nivel nacional antes de la reforma. Para la repartición proporcional se aplica el método Sainte Laguë/Schepers, sin compensar ya a nivel de las circunscripciones plurinominales posibles escaños excedentes (Überbangmandate). Este primer cálculo de conversión de votos en escaños determina el número mínimo de escaños al que tiene derecho un partido a este nivel plurinominal, su suma por partido de todas las circunscripciones plurinominales indica así el «derecho a escaños» (Sitzanspruch) de los partidos a nivel nacional.

Segundo, la proporcionalidad se perfecciona a nivel de la Federación en base a la suma de los votos que han recibido los diferentes partidos en todo el país, aplicando de nuevo el método Sainte-Laguë/Schepers. A diferencia del arreglo anterior a la reforma, se compensan ahora los escaños excedentes. Además se trata de integrar el «derecho a escaños» de los partidos en la proporcionalidad entre votos y escaños a nivel nacional para lo que se aumenta el número de los escaños del Bundestag hasta que se consigue la más exacta proporcionalidad. El número total de escaños del Bundestag se determina prácticamente con base al partido político que demuestra la más alta cantidad de «derecho a escaños» en relación a los escaños que hubiera recibido con base a la proporcionalidad nacional. Cuanto más alta la diferencia, que emana de la adaptación de los diferentes puntos de referencia en los procesos de distribución de los escaños (primero a los Laender: población, segundo a los partidos: votos), mayor es el aumento de escaños para compensarla proporcionalmente. Así, en las elecciones de 2013, el número de escaños del Bundestag aumentó de 598 a 630, en las elecciones de 2017 a 709.

\section{El sistema electoral alemán reformado en su dimensión de modelo}

Es interesante observar que el interés por el sistema alemán se cultiva tanto en países que aplican un sistema de representación por mayoría, pues piensan en el criterio de la proporcionalidad que el sistema alemán combina con el voto personal, como en países que usan un sistema proporcional que piensan en el criterio de la participación (como fue definido más arriba) que el sistema alemán combina con la proporcionalidad. Es en estas virtudes que se fundamenta precisamente lo modélico del sistema alemán cuando se refieren a él en los debates sobre reforma electoral en el mundo ${ }^{20}$.

20 Report of the Royal Commission on the Electoral System: Towards a Better Democracy, V.R. WARD, Govt. Printer, Wellington, N.Z., 1986; Shugart, M.S., Wattenberg, M., Mixed-Member Electoral 
La pregunta es, si el sistema electoral alemán reformado conserva su atractivo como modelo. A este respecto tengo serias dudas. Con la reforma de 2013 , el sistema electoral alemán ha mantenido varios elementos claves que se refieren, por ejemplo, a la combinación del voto unipersonal con el pluripersonal (de lista), del principio de decisión mayoritario con el proporcional, todo dentro de un sistema de representación proporcional. Sin embargo, con la reforma se ha agudizado el carácter proporcional del sistema, convirtiéndolo en uno de tipo compensatorio por medio de la compensación no solo de los escaños excedentes, sino también de las desigualdades en el grado de proporcionalidad entre votos y escaños de los partidos que sobrepasaron la barrera legal del cinco por ciento. Los efectos no-deseados de la reforma han resultado peores que las fallas que se querían resolver. El primer efecto negativo es la extensión numérica del Bundestag, cuya cantidad de miembros, por lo demás, ha resultado totalmente dependiente del sistema de partidos y del comportamiento electoral. Es el mayor objeto de crítica por ser el efecto más visible e incómodo, que de verdad era previsible. Luego, con la reforma de 2013 se perdió una de las virtudes del sistema original, la sencillez. El cálculo de la proporcionalidad es extremamente complejo, y casi imposible de entender con base en el texto legal. Nadie capta fácilmente el mecanismo de compensación, ni entiende por qué el partido más votado y con escaños excedentes puede beneficiarse con más escaños en el proceso de compensación proporcional a nivel nacional. Y finalmente: el sistema reformado cuenta con poca legitimidad. La reforma consumió el modelo. Por todos lados se pide una urgente reforma ${ }^{21}$, incluso por parte de los partidos que se beneficiaron con una compensación proporcional a ultranza. Hasta los propios inspiradores matemáticos se sumaron a la crítica ${ }^{22}$. No obstante, pensar en el sistema alemán para reformas electorales no significa extenderlo a mecanismos técnicos que ni convencen por sí mismos. Convendría recurrir al espíritu de la representación proporcional personalizada.

Systems: The Best of Both Worlds?, op. cit.; Vidal Prado, C., El sistema electoral alemán y su posible implantación en España, Tirant lo Blanch, Valencia. 2012.

21 Por ejemplo Dehmel, N., Jesse, E., «Das neue Wahlgesetz zur Bundestagswahl 2013. Eine Reform der Reform der Reform ist unvermeidlich» [La nueva ley electoral para la elección del Bundestag de 2013. Una reforma de la reforma de la reforma es imprescindible], en: Zeitschrift für Parlamentsfragen 44, 2013.; BulL, H.D., «Erfolgswertgleichheit — eine Fehlkonstruktion im deutschen Wahlrecht» [La igualdad de valor de logro - una construcción en falso en el sistema electoral alemán], en: DVBl. (Deutsches Verwaltungsblatt) 129, 2014; Grotz, F., «Happy End oder endloses Drama? Die Reform des Bundestagswahlsystems» [Happy end o drama sin fin. La reforma del sistema electoral del Bundestag], en: Jesse, E. /STURM, R. (Eds.): Bundestagswahl 2013. Voraussetzungen, Ergebnisse, Folgen [La elección al Bundestag de 2013. Condiciones preliminares, resultados, consecuencias], Nomos, Baden-Baden, 2014.

22 Hablaron de un sistema «imperfecto» (Pukelsheim/ Rossi, 2013), profesando la falsa idea de que podría existir un sistema perfecto, independiente del contexto, de valores, intereses, estructuras y experiencias. Una variante de esta postura descontextualizada es la tradicional búsqueda por el mejor sistema electoral: Lijphart/ Grofman, 1984; Duverger, 1984; Sartori, 1994; Shugart, M.S., Wattenberg, M., Mixed-Member Electoral Systems: op. cit.; y otros que dista de mi enfoque histórico-empírico (Nohlen, 2003). 


\section{LA INJERENCIA DEL TRIBUNAL CONSTITUCIONAL FEDERAL EN MATERIA DE REFORMA ELECTORAL}

En la República Federal de Alemania, la cuestión del sistema electoral ha pasado de ser una cuestión de teoría política, enfocada en saber cuál es el mejor sistema para el desarrollo de la democracia en un determinado país, y una cuestión de poder, decidida políticamente en consonancia con las mayorías parlamentarias, a una cuestión de derecho, de jurisdicción constitucional. El Tribunal Constitucional Federal (TCF) se adjudica la última palabra en relación al sistema electoral. Ha extendido su poder hacia el propio proceso legislativo.

En la Ley Fundamental no se menciona el sistema electoral. Esta ausencia en la Constitución sugiere que el sistema electoral en su determinación corresponde al proceso político, la que cae en el ámbito de legítimas controversias políticas. En caso de que el legislador opte por un sistema proporcional, la restricción constitucional también sugiere que el principio de igualdad electoral, consagrado en el artículo 38 de la Ley Fundamental, «no debería ser aplicado en el sentido del principio de representación proporcional, (sino, en concreto): igualdad del valor numérico de los votos sí, igualdad del valor de logro, no» ${ }^{23}$. Sin embargo, el TCF profesa una estricta interpretación de la igualdad del sufragio en sentido de igualdad del valor de logro de los votos. Dado que este tribunal, por medio del recurso de inconstitucionalidad a menudo está llamado a sentenciar en materia de sistema electoral, verifica, de hecho, si el principio de igualdad del sufragio en el sentido de la igualdad del valor de logro de los votos ha sido respetado dentro del sistema de representación proporcional personalizada, Así, el debate de los últimos años sobre el sistema electoral en Alemania fue convertido en una cuestión legal-jurisdiccional por una decisión del Tribunal Federal Constitucional.

Con la ley del 9 de mayo de 2013, el Bundestag acordó otra reforma electoral, esta vez con una amplia mayoría, que obedecía al máximo a los criterios y las resoluciones del Tribunal Constitucional Federal. Esta reforma parece marcar el fin de un muy problemático proceso, en el que el Bundestag, legitimado en su labor legislativa por el pueblo, perdió la supremacía en un tema institucional

23 Frowein, J.A., «Rechtsgutachten zu der Vereinbarkeit der Verhältniswahl in kleinen Wahlkreisen (Dreier- oder Vierer-Wahlkreisen) mit dem Grundgesetz» [Dictamen jurídico sobre la compatibilidad de la representación proporcional en circunscripciones pequeñas (circunscripciones de tres o cuatro escaños) con la Ley Fundamental], en: Bundesministerium des Innern: Rechtsgutachten zu der Vereinbarkeit der Verbältniswabl in kleinen Wablkreisen (Dreierwablkreisen) mit dem Grundgesetz, Bonn, 1968, p. 12.

La relación entre el valor de logro y el valor numérico de los votos es, a menudo, mal entendida. La igualdad del valor de logro de los votos presupone la igualdad del valor numérico de los votos, pero no es su consecuencia automática. Por el contrario, el valor de logro varía de acuerdo a los sistemas electorales. En caso de desigualdades observadas, consideradas relevantes bajo aspectos de la igualdad ante la ley, es necesario explorar si estas se deben al sistema electoral o a otras disposiciones legales o a factores que ya causan desigualdades en el valor numérico de los votos. Sólo si el sistema electoral es la causa, se trata, en contraste con la desigualdad en el valor numérico, de desigualdad en el valor de logro de los votos. 
clave: con qué sistema electoral elegirse a sí mismo. Entre noviembre de 2011 y febrero de 2014 el Tribunal le quitó también al Bundestag la capacidad de definir el sistema electoral para la elección del contingente alemán al Parlamento Europeo. En síntesis, en pocos años se ha cambiado todo el cuadro político-jurídico de los sistemas electorales en Alemania. La política de la reforma electoral la marca el Tribunal Constitucional Federal.

\section{La doctrina proporcionalista del Tribunal Constitucional Federal}

La actividad jurisdiccional del TCF en materia electoral se basa en la comprensión de la igualdad en el sufragio como caso de aplicación del principio general de igualdad, tal como lo establece el artículo tercero de la Ley Fundamental. Se juntan, además, otros dos supuestos: la aplicabilidad de los principios del sufragio en el ámbito de los sistemas electorales y la idea de que la representación proporcional de la República Federal tiene que orientarse al ideal de proporcionalidad mostrada en votos en la composición del Bundestag.

En cuanto al primer supuesto, es preciso recordar que el principio jurídico de igualdad del sufragio estaba dirigido históricamente contra todas las desigualdades en el peso del voto de los individuos con derecho de sufragio, graduado de acuerdo a diferentes criterios (por ejemplo propiedad, educación, mérito) así como en contra de la desigualdad de la llave de la representación, es decir, en contra de una desigualdad en el número de habitantes o personas con derecho de sufragio de las circunscripciones electorales, representadas por un diputado. Ambos requisitos de igualdad tocan el derecho de sufragio. Más tarde, se desarrolló la comprensión de la igualdad de sufragio como representación proporcional de todos los votos en la asamblea por elegir. Este requisito de igualdad toca el sistema electoral. Esta necesaria diferenciación entre las áreas temáticas a las que se aplica el principio de igualdad, se pierde fácilmente de vista, más aún, cuando como en la práctica idiomática alemana se usa derecho de sufragio y sistema electoral de manera sinónima por medio de un único concepto: derecho electoral (Wablrecht). Pero hay que distinguir estrictamente entre el sufragio, el derecho a elegir y ser elegido, por un lado, y el sistema electoral, los mecanismos de entrega de los votos y de su conversión (en las elecciones parlamentarias) en escaños, por el otro. Por el derecho de sufragio rigen disposiciones uniformes de naturaleza constitucional que se aplican en todas las democracias representativas: el derecho de sufragio tiene que ser universal, igual, directo y secreto, para que pueda ser considerado como democrático. Está sujeto en todas las democracias constitucionales a examen jurídico continuo. En este sentido, las cuestiones de sufragio son asuntos jurídicos. Para el sistema electoral, no existen normativas uniformes. El significado y la función de los sistemas electorales, por el contrario, consisten en las diferencias que hacen mecánica- y psicológicamente en la formación de las preferencias políticas y en la transmisión de estas en escaños parlamentarios. Se 
ofrecen alternativas entre diferentes sistemas electorales, y las opciones nacionales dependen de las constelaciones de poder, los efectos de los sistemas electorales de determinados contextos. A diferencia del caso del sufragio, las disposiciones constitucionales son más bien escasas. Pocas constituciones prescriben el sistema electoral parlamentario. Si este es el caso, entonces, por supuesto, los tribunales constitucionales pueden ser llamados a revisar directamente la aplicación constitucional del sistema electoral por parte de las autoridades electorales ${ }^{24}$.

En cuanto al segundo supuesto, el TCF aplica los principios del sufragio y el principio general de la igualdad de la Ley Fundamental al sistema electoral de manera que en el sistema de la representación proporcional personalizada tenga que garantizarse no sólo el valor numérico de los votos, sino también su valor de logro. Esta postura también es producto de cómo el TCF interpreta el sistema electoral para el Bundestag alemán. El Tribunal reconoce que el valor de logro de los votos varía en función de los sistemas electorales que requieren jurídicamente una evaluación diferente. Mientras que en sistemas de representación por mayoría la igualdad en el valor de logro de los votos sería ajeno al sistema y, por tanto, no podría ser un parámetro de evaluación jurídica-legal, sería esencial en sistemas de representación proporcional, porque el objetivo de la representación proporcional consistiría en la igualdad del valor de logro. Cada voto tendría que contribuir de manera igual a la composición del Bundestag. Sin embargo, restricciones al principio de la representación proporcional podrían ser compatibles con la Ley Fundamental si se les pudieran justificar funcionalmente. Así, el TCF ha reconocido a la barrera de cinco por ciento como constitucionalmente compatible debido a su función de estabilizar las relaciones del gobierno, así como también a los escaños excedentes, sin compensación proporcional, para posibilitar en la práctica la combinación de la elección uninominal y de la lista pluripersonal (en opinión del Tribunal: de la representación por mayoría y de la representación proporcional). Sin embargo, su número debería ser limitado. En su decisión del 25 de julio de 2012, el TCF había fijado un techo de 15 escaños excedentes sin compensación proporcional. Por lo tanto, la clasificación del sistema electoral alemán por parte del TCF como proporcional legitima la aplicación del criterio de la igualdad del valor de logro de los votos. Sólo en segundo lugar el Tribunal reconoce límites a este principio, fundamentados en razones funcionales y en una medida aún compatible con la Constitución.

Así, el TCF se encuentra llamado continuamente a resolver disputas políticas sobre el sistema electoral y decidir sobre su compatibilidad con la Ley Fundamental, tomando como criterio el sufragio igualitario en la forma de la igualdad

24 Este examen se llevó a cabo, por ejemplo en el caso de la Sala del Tribunal Supremo de Justicia de Venezuela, responsable de Asuntos Constitucionales, cuando las autoridades electorales permitieron que la representación proporcional personalizada, introducida en 1989 acorde con el modelo alemán, se aplicase en las elecciones de 2005 a la manera de un sistema segmentado; para más detalles véase NoHLEN / NoHLEN (2007). 
de valor de logro de los votos. Ya el razonamiento del Tribunal en su sentencia sobre el peso negativo del voto del 3 de julio de 2008 me hizo hablar de un «Volver a Weimar» ${ }^{25}$, dado que decía: «El objetivo de la representación proporcional es que todos los partidos estén representados en el órgano a ser elegido en una relación lo más aproximada posible al número de votos recibidos». Es un retorno a la idea de una representación proporcional pura. Weimar, sin embargo, estaba incluso presente de manera mucho más profunda en la medida en que el TCF hacía propia la jurisdicción de la Corte Suprema del Estado (Staatsgerichtshof) de aquella República, según la «que básicamente comprende la igualdad del sufragio en un sistema de representación proporcional, no sólo la igualdad en el valor numérico, sino también la igualdad en el valor de logro» ${ }^{26}$. Entre los constitucionalistas lo defendió también y con fervor Hans Meyer sin sustento en experiencias fuera de Alemania cuando apuntaba: «Igualdad del sufragio requiere siempre el valor de logro igualitario de los votos» ${ }^{27}$, y en contra de la argumentación jurídica más abierta, dibujada por Jochen Abr. Frowein y Roman Herzog ${ }^{28}$. Con su doctrina, el Tribunal abrió, además, la puerta hacia una mayor influencia de los matemáticos en la consultoría política como protagonistas de procedimientos de escrutinio en su teoría de orden puramente matemático, buscando la mejor correspondencia proporcional entre votos y escaños (ver especialmente Pukelsheim, 2000 y 2004). Esta confluencia de ideas y medios hizo que la ciencia política perdiera la influencia que ejercía en anteriores debates en los sesenta sobre reformas electorales ${ }^{29}$ al igual que el pensamiento crítico al TCF, que provenía más tarde de la jurisprudencia misma, aunque se articulaba de forma muy clara. Christofer Lenz, por ejemplo, afirmó: «La igualdad del sufragio nunca postula la igualdad del valor de logro de los votos, pero siempre la garantía de igualdad de la posibilidad de éxito ${ }^{30}$ ». Este pensamiento quedó marginado.

\section{El valor de logro en el derecho público comparado}

El TCF ejerce la práctica de pedir estudios de derecho comparado antes de tomar decisiones ${ }^{31}$. La pregunta es, hasta qué punto se deja influir en sus sentencias por soluciones exteriores. Con respecto a la igualdad de sufragio es lógico hacer la comparación con los países de habla alemana, dado que se puede suponer una cierta cultura jurídica común.

\footnotetext{
25 Nohlen, 2009

26 Misch, 1974: 208.

27 Hans Meyer (1973: 113 y ss., 120 ss., y 134 ss.)

28 Jochen Abr. Frowein y Roman Herzog (1968).

29 Ver Vogel/ Nohlen/ Schultze, 1971.

30 Christofer Lenz (1996: p. 356).

31 Véase por ejemplo MPI, 1997.
} 
En contraste con Alemania, en Suiza el sistema electoral está consagrado en la Constitución. Según su artículo 73, las elecciones al Consejo Nacional se adjudican «de acuerdo con el principio de proporcionalidad». Rige la representación proporcional en circunscripciones plurinominales y uninominales. La delimitación de las circunscripciones también está consagrada en la Constitución Federal. Cada cantón y medio-cantón constituye una circunscripción electoral. La adjudicación de los escaños a las circunscripciones se realiza de acuerdo a la «población residente» por el método Hare y residuo más grande. Pero cada circunscripción tendrá derecho a por lo menos un asiento ${ }^{32}$. No hay compensación nacional. La ley electoral no garantiza ni la igualdad del valor numérico ni la igualdad del valor de logro de los votos. Es de destacar la particularidad de que en Suiza, tradicionalmente orientada hacia la proporcionalidad (Lehmbruch, 1967) no se problematiza en absoluto la desigualdad de sufragio ${ }^{33}$.

En Austria, la Constitución se expresa sobre el sistema electoral. Según el Art. 26, apartado 2, las elecciones al Consejo Nacional tienen lugar «de acuerdo con los principios de representación proporcional». El parlamento es elegido por representación proporcional en circunscripciones con compensación proporcional a nivel nacional. La asignación de escaños a los partidos se llevará a cabo en tres niveles: en primer lugar, a nivel regional (43 circunscripciones), a continuación, en el plano de los Estados federados ( 9 circunscripciones) y, finalmente, en el ámbito de la Federación. ${ }^{34}$ En la adjudicación de los escaños a nivel de los Estados federados y de la Federación sólo pueden participar los partidos que han ganado ya sea un mandato a nivel de circunscripción regional o $4 \%$ de los votos a nivel federal. Para la asignación de escaños de manera proporcional es decisivo el nivel federal-nacional. Se aplica el método d'Hondt ${ }^{35}$.

A pesar de todas las diferencias de detalle, en la función de la asignación nacional de votos el sistema electoral de Austria es idéntico al alemán. En este sentido, la jurisprudencia de la Corte Constitucional de Austria en relación a la igualdad del sufragio es particularmente relevante. A diferencia del TCF, la Corte austríaca

32 El votante tiene tantos votos como diputados por elegir. En las circunscripciones plurinominales, los escaños se asignan de acuerdo con el método de Hagenbach-Bischoff.

33 Véase Garrone, 1991: 156 sigs.

Dada la fragmentación de los partidos, los resultados electorales son, por cierto, relativamente proporcionales. En las elecciones nacionales de 2007, con el 28,6\% de los votos, el SVP logró el 31,0\% de los escaños. Para los partidos de tamaño decreciente se dio la siguiente relación: SPS: 19,3\% y 21,5\%; FDP: $15,5 \%$ y $15,5 \%$; CVP: $14,3 \%$ y $15,5 \%$; GPS: $9,5 \%$ y 10,0\% (datos de Linder et al., 2010).

34 Los 183 escaños se asignan de acuerdo con el número de ciudadanos en las circunscripciones regionales cuyo tamaño varía de entre uno y ocho escaños. A nivel de los Estados federados, el tamaño de las circunscripciones varía entre 7 y 36 escaños.

35 De hecho surgen resultados electorales muy proporcionales. Por ejemplo, en las elecciones del año 2008, el Partido Social Demócrata con el 29,3\% de los votos consiguió el 31,1\% de los escaños, el Partido Popular Austríaco (ÖVP) con el $26,0 \%$ de los votos $27,9 \%$ de los escaños, el Partido de la Libertad, con $17,5 \%$ de los votos el 18,6\% de los escaños, los Verdes con 10,4\% de los votos el 10,9\% de los escaños (datos según Poier, 2010: 217 y 220). 
redujo el principio de igualdad del sufragio tradicionalmente a la igualdad del valor numérico de los votos: «Pedir, además, que cada voto tenga el mismo peso, el mismo beneficio o valor de logro, queda fuera del principio de igualdad de sufragio, fuera incluso del reino de la posibilidad $»^{36}$. La Corte Constitucional austríaca también explica por qué diferencia en el marco de la garantía constitucional de la igualdad de sufragio entre el valor numérico y el valor de logro de los votos. «El principio de la igualdad del sufragio tiene significado y efecto sólo en el procedimiento de votación, donde cada voto válido debe tener el mismo valor numérico. Qué valor tienen los votos en la distribución de los escaños depende de circunstancias cambiantes y contingentes» ${ }^{37}$. La Corte reafirmó su punto de vista tradicional en 1959: «Es imposible escrutar los votos, entregados con igualdad en el valor numérico, de manera que tengan un valor de logro equivalente. El efecto del sufragio igualitario, por tanto, se agota con el recuento de los votos emitidos. El principio de la igualdad del sufragio no tiene ningún efecto sobre los factores que determinan el valor de logro de los votos.... ${ }^{38}$. «El principio de la igualdad del sufragio, por lo tanto, significa simplemente una igualdad del peso de los votos ex ante (de la votación) (....), cuestiones del peso del voto ex post (de la votación) y por lo tanto cuestiones referidas al valor de logro de los votos no son cuestiones de la igualdad del sufragio, sino tienen que ser tratados como problemas de la representación proporcional y de las variantes constitucionalmente permisibles» ${ }^{39}$. En el dictamen preparado para el TCF por parte del MPI, incluso se llegó a la conclusión de que en Austria «en principio (...) la restricción de la igualdad de valor de logro es legitimada por la propia Constitución y por lo tanto la consideró constitucional» ${ }^{40}$.

\section{Objeciones a la postura proporcionalista del Tribunal Constitucional Federal}

En contra de esta interpretación del sistema electoral alemán en términos de una aplicación estricta del principio de igualdad del valor de logro hay varios argumentos de muy diferente índole que trataremos brevemente a continuación.

Primero, la representación proporcional debe entenderse no sólo como un principio en el sentido de principio de representación, sino también — como ya se ha dicho- como una regla de decisión. Si la representación proporcional no está consagrada en la Constitución, puede simplemente actuar como regla general de cómo otorgar los escaños a los partidos o candidatos en circunscripciones

36 Colección de la Corte Constitucional de Austria (VfSlg) 1.381 / 1931, 227, citado en PoIER, 2001: 241.

37 Véase, ibíd., 216, 227, citado en MPI (1997: 664).

38 Véanse, 3653/1959, 467, 469, cit. en MPI (1997: 664)

39 POIER, 2001: 245

40 MPI, 1997: 665 
plurinominales acorde con las partes correspondientes de los votos. Este es el caso, por ejemplo, de la representación proporcional en circunscripciones pequeñas hasta medianas. Se aplica la regla decisoria proporcional. El sistema de circunscripciones electorales trinominales es incluso considerado como mayoritario.

Segundo, la representación proporcional como principio no puede ser entendida como entidad matemática, sino como parámetro orientador general de la representación política: «the proportionality principle should not be cast in stone as an exact mathematical requisite... It should rather serve as a general guideline with multifarious possible applications in different contexts ${ }^{41}$. Este punto de vista se refleja empíricamente a nivel internacional en sistemas de representación proporcional que muestran una alta diversidad en la igualdad de valor de logro de los votos ${ }^{42}$, sin que esto, en otro lugar, haya sido considerado constitucionalmente cuestionable.

Tercero, conviene reflexionar sobre el concepto de «lo más posible» en la descripción del principio de proporcionalidad en el marco de la representación proporcional: la proporción de escaños de un partido debe corresponder lo más posible a su proporción de votos, no es una exhortación a realizarla lo más estricta (como máxima) posible, sino una relativización del principio. El «más» se refiere a las condiciones que deben tenerse en cuenta, las que podrían limitar la aplicación del principio puro.

Cuarto, en materia de sistemas electorales es cuestionable la idea de que se trate de imponer un principio único. Más bien, es de reconocer que los sistemas electorales pueden perseguir distintos objetivos y que el requisito sería equilibrar estos, incluso y especialmente cuando domina el principio de representación proporcional $^{43}$. El sistema electoral para el Bundestag alemán es un buen ejemplo, porque este de hecho trata de realizar diferentes funciones y objetivos simultáneamente, lo que sólo puede lograrse si ninguno de ellos se utiliza en su máxima expresión. Generalmente, la diversidad de los sistemas de representación proporcional en el mundo, permite suponer que se busca en cada caso el respectivo equilibrio nacional entre las funciones. Al mismo tiempo afirma la validez de la multifuncionalidad de los sistemas de representación proporcional.

Quinto, la igualdad del valor de logro no puede ser establecida por ley. El grado de igualdad de este valor depende empíricamente del sistema de partidos y del comportamiento electoral. Aquí se puede observar una relación paradójica: entre más exacto el posible grado de la proporcionalidad gracias a la ley, menor puede resultar la igualdad del valor de logro empíricamente, ya que los votantes se equivocan al suponer que ahora cada partido pequeño tiene la oportunidad de entrar en el Parlamento, lo que no ocurre por razones matemáticas. En este sentido, también en un sistema de representación proporcional pura, muchos votos

41 Brighouse / Fleurbaey, 2010: p. 145.

42 Ver Nohlen/Stöver, 2010.

43 Ver Nohlen, 2014a: p. 229. 
se pierden, no cuentan. Esto enseña la comparación de los resultados electorales en sistemas de representación proporcional con y sin barrera legal.

Sexto, ni el principio de representación por mayoría, ni el principio de representación proporcional son políticamente neutrales. Detrás de ellos se esconden intereses político-electorales. El postulado de la igualdad del valor de logro proviene generalmente de partidos pequeños o minoritarios, a los que también les gusta servirse del argumento de la justicia electoral, como si sólo la proporcionalidad exacta fuese justa. Este argumento, sin embargo, está referido sólo a la representación parlamentaria, donde puede convencer fácilmente. Su reduccionismo sólo será reconocido, cuando se suma y expande la idea de proporcionalidad a la esfera del gobierno. ¿Es justo? hay que preguntarse, si los partidos pequeños en los gobiernos de coalición, que son una consecuencia casi inevitable de la representación proporcional con un alto grado de proporcionalidad, adquieren una influencia en las decisiones políticas a favor de sus clientelas, lo que supera ampliamente el peso real de estos partidos en el electorado.

Séptimo, en las elecciones parlamentarias se elige no sólo el Parlamento, sino inclusive también el Gobierno — cuando lo permite el sistema de partidosMientras más proporcional es la representación, entonces resulta más fragmentado el sistema de partidos y menores posibilidades de los electores de escoger directamente entre los candidatos a primer ministro y equipos de gobierno. Así, las decisiones sobre la formación de coaliciones se tomarán después de las elecciones por los partidos. Mientras mayor sea la igualdad en el valor de logro y la consideración de cada voto individual, entonces menor es el poder de todos los electores juntos.

Octavo, la aprobación de la representación proporcional en el electorado es dependiente de la explicación de los posibles efectos que se relacionen con ella conforme a toda nuestra experiencia. Incluso en el Reino Unido, donde los terceros partidos son perjudicados enormemente por el sistema de mayoría relativa, y donde algunas comisiones de reforma ya han recomendado una reforma electoral, la idea proporcional sólo recibe un apoyo mayoritario en la opinión pública, cuando se evita discutir ampliamente las posibles consecuencias de la representación proporcional. No es de extrañar que el electorado británico ha rechazado, después de una adecuada información, incluso la prevista introducción del voto alternativo, un sistema a medio camino hacia le representación proporcional, en el referéndum de mayo de 2011.

En suma, las preocupaciones planteadas aconsejan no sobrevalorar el principio de representación proporcional en la evaluación de los sistemas electorales, cuando el legislador se ha pronunciado por él constitucional o legalmente, sino de aplicarlo con moderación y mesura.

\section{Hacia la judicialización de la política institucional}

Por medio de su doctrina en cuestiones de sistemas electorales proporcionales, el TCF entró de lleno en competencia política con el órgano constitucional 
legitimado democráticamente, el Bundestag. Su argumentación en síntesis: dado que los sistemas electorales afectan a las condiciones de la competencia política, la mayoría parlamentaria, por tanto, decidiría en cierto sentido en su propia causa. En el caso de la legislación electoral existiría el peligro de que la respectiva mayoría parlamentaria se dejara guiar por el objetivo de mantenerse en el poder en vez de tener en cuenta el bienestar público. Por lo tanto, el diseño de la ley electoral tendría que ser sujeto de un estricto control judicial constitucional. Con esta confianza en sí mismo y desconfianza en los partidos políticos y en el orden de competencias constitucionales de la democracia representativa legitima de verdad la transferencia de decisiones políticas a la instancia judicial, acorde con una tendencia que se extiende más allá de Alemania: la judicialización de la política. Los argumentos expuestos sirvieron de base de la sentencia del TCF del 9 de noviembre de 2011 en relación al sistema electoral para el contingente alemán del Parlamento Europeo. Se canceló la barrera legal del cinco por ciento. Cuando el Bundestag legisló una barrera legal del tres por ciento, el TCF la suprimió también. Así, la cuestión del sistema electoral se estrechó a una cuestión jurídica ${ }^{44}$. Ha desplazado en importancia al sistema electoral como asunto de teoría política y de poder.

El TCF ciertamente no está actuando por propia iniciativa. Sin embargo, se lo involucra en disputas político-jurídicas por parte de actores políticos que no han podido imponerse en el proceso político. En materia electoral, el Tribunal no sólo comprueba la compatibilidad de la ley electoral con la Constitución, decidirá también en favor de cualquiera de las partes en disputa. A veces se compara el Tribunal con una especie de cámara legislativa adicional, dando por entender con esto que su actuación no es tan apolítica, orientada solo en el beneficio de todos, como pretende ser. El grupo minoritario en el proceso parlamentario democrático se sirve a menudo de la jurisdicción constitucional del TCF para prevalecer finalmente, de acuerdo con la estrategia política que se expresa en el lema «Nos vamos a ver de nuevo en Karlsruhe» (la sede del TCF) ${ }^{45}$. La disputa termina en una cuestión de poder entre

44 En la discusión de mi charla «La representación proporcional personalizada en perspectiva comparada» en el Simposio «El sistema electoral para el Bundestag - La cuadratura del círculo» de la sección alemana de la Comisión Internacional de Juristas (Berlín, junio de 2013) el diputado Dieter Wiefelspütz me señaló, que los sistemas electorales son derecho (o sea ley). Por supuesto, los sistemas electorales, una vez legislados, son ley (derecho). Mi tesis, sin embargo, no trata de estos hechos innegables, sino del diseño de los sistemas electorales y qué aspectos, factores e instituciones influyen en este proceso, distinguiendo de forma gruesa entre factores de teoría política, de poder y de derecho.

45 Se tiene que tomar en cuenta cuán político puede ser el argumento en la controversia de los partidos, también el de los expertos consultados. Por ejemplo, cuando la asignación de los escaños por la representación proporcional en circunscripciones plurinominales y la compensación proporcional a nivel nacional en la ley electoral de 25 de noviembre de 2011 fue descrita por Hans Meyer como «la pieza de sistema electoral más descuidada que he visto nunca» (Citado en Der Spiegel, 22/2012. 34). Esta polémica muestra únicamente que este jurista desconoce por completo el panorama internacional de los sistemas electorales, específicamente los sistemas proporcionales nórdicos, o sea demuestra, cómo un experto descuida estándares de la sistemática electoral en función de representar, como abogado, intereses partidistas ante el Tribunal Constitucional Federal. Esto no es responsabilidad del Tribunal mismo, sin embargo, dice mucho acerca de la dimensión política del conflicto dentro del procedimiento jurisdiccional. 
los órganos constitucionales, en la que el TCF, como destacó Matthias Jestaedt cuenta «con el poder de la última palabra, no reversible por otras instancias» ${ }^{46}$. El poderoso TCF se encuentra en peligro de reducir indebidamente la libertad política del órgano legislativo, democráticamente legitimado, en asuntos del sistema electoral. Legislar sin legitimidad democrática contradice la democracia.

No obstante, el TCF sentencia acorde al procedimiento judicial, interpretando la constitución y/o la ley. Así, el TCF interpreta el sistema electoral al Bundestag de manera que el principio de representación proporcional, que no está consagrado en la Ley Fundamental, se aplica como medida a su evaluación jurídica por medio del principio de la igualdad de sufragio en el sentido de la igualdad de valor de logro de los votos, derivado de él. En este quehacer de interpretación, por un lado no se respetan lo suficiente las limitaciones significativas a este principio, provenientes de la teoría de la democracia y de experiencias empíricas científicamente comprobadas, por otro lado se menosprecian las distinciones necesarias, provenientes de la sistemática electoral, entre diferentes tipos de sistemas de representación proporcional, entre ellos la representación proporcional personalizada con barrera legal y su propia lógica sistémica y contextual. O sea, las sentencias del TCF no son capaces de convencer ni desde el punto de vista puramente jurídico, ni desde la perspectiva comparada de derecho público, entonces la cuestión jurídica en materia de sistema electoral apenas se puede distinguir de la cuestión de poder ${ }^{47}$.

\section{CONCLUSIONES}

Al inicio de nuestro análisis hemos afrontado la coexistencia de diferentes comprensiones y clasificaciones del sistema electoral alemán para promover una comprensión y una clasificación uniformes. Como dice su término en alemán: es un sistema proporcional personalizado. Lo hemos comprobado a través de su génesis, sus reformas, las decisiones jurisdiccionales al respecto y los efectos del sistema ejercidos sobre el sistema de partidos políticos. Es la comprensión del sistema por parte del TCF, la que es fundamental para su jurisdicción. La insistencia del Tribunal en

46 Jestaedt, M., «Phänomen Bundesverfassungsgericht. Was das Gericht zu dem macht, was es ist» [El fenómeno del Tribunal Constitucional Federal. Lo que hace que el Tribunal sea lo que es, en: JESTAEDT, M. / Lepsius, O. / Möllers, CH. / Schönberger, CH.: Das entgrenzte Gericht. Eine kritische Bilanz nach sechzig Jahren Bundesverfassungsgericht [El Tribunal sin límites. Un balance crítico de sesenta años del Tribunal Constitucional Federal]. Suhrkamp, Francfort del Meno, 2011, p. 81.

47 En la jurisdicción constitucional comparada en materia de los sistemas electorales, se observa incluso un cierto localismo y mucha ingeniosidad, por decir lo menos. Por ejemplo, la Corte Constitucional de Renania del Norte / Westfalia, en diciembre de 2019, ha declarado inconstitucional el sistema de mayoría relativa con el argumento de que con este sistema una minoría podría ganar una elección. Solo la mayoría absoluta de los votos cumpliría con el criterio mayoritario (véase Frankfurter Allgemeine Zeitung, 23.12.2019:11). Qué desconocimiento de la historia del principio mayoritario, de la tradición conceptual surgida en la edad media y consustancial con el parlamentarismo y (más tarde) con la democracia, testimoniado por el caso inglés. Mayoría es mayoría, relativa o absoluta. La Corte Constitucional de este Land alemán dictó una sentencia de contenido antidemocrático. 
que el sistema proporcional personalizado tenga que orientarse en alcanzar el valor de logro de los votos, es la incuestionable idea del sistema y no permite otra comprensión. Sin embargo, con la reforma de 2013, se abre otra distinción. Tratando de adecuar el sistema electoral a la doctrina del TCF, el sistema alemán ha cambiado y conviene diferenciar entre el anterior y el posterior a la reforma. En términos generales, con la reforma, el sistema electoral alemán ha perdido algunas de sus virtudes, especialmente en transparencia y legitimidad, lo que le ha vuelto menos atractivo para que sistemas electorales de otros países que piensan en su reforma puedan orientarse en él. El nuevo sistema ha generado enormes críticas en nuestro país desde diferentes perspectivas. En la misma jurisprudencia se habló de una Feblkonstruktion (construcción en falso ${ }^{48}$ ). Desde la ciencia política, ya en uno de los primeros comentarios se sostuvo que una reforma de la reforma era imprescindible ${ }^{49}$. Sin embargo, tras haber llegado - en contra de lo que era la voluntad de los padres de la Ley Fundamental - a una representación proporcional (casi) pura y a la fragmentación del sistema de partidos tipo Weimar, dificulta plantear y alcanzar una reforma, sobre todo cuando el TCF ha elevado a requisito la existencia de un alto consenso entre los partidos políticos. La necesaria reforma se está haciendo esperar. Como de costumbre, opiniones menos fundadas señalan a los partidos como los supuestos causantes del «drama sin fin» ${ }^{50}$. Y casi nadie se atreve a plantear como problema el desafortunado rol del prestigioso TCF que ha asumido competencias que no le corresponden a nuestro juicio en una democracia representativa y sin accountability, sin tener que responder democráticamente por sus desmesuradas decisiones.

$$
* * *
$$

TITLE: The reform of the german electoral system and the Federal Constitutional Court

AвSTRACT: This paper analyzes the German electoral system, its reforms and the doctrine of the German Federal Constitutional Court.

RESUMEN: Se analiza en este trabajo el sistema electoral alemán, las reformas sufridas y la doctrina del Tribunal Constitucional federal alemán.

KEY wORDS: Electoral system. Constitutional Court

Palabras Clave: Sistema electoral. Tribunal Constitucional

FECHA DE RECEPCIÓN: 24.01.2020

FECHA DE ACEPTACIÓN: 19.02.2020

48 Bull, H.D., «Erfolgswertgleichheit — eine Fehlkonstruktion im deutschen Wahlrecht» [La igualdad de valor de logro — una construcción en falso en el sistema electoral alemán], en: DVBl. (Deutsches Verwaltungsblatt) 129, 2014.

49 Dehmel, N., Jesse, E., «Das neue Wahlgesetz zur Bundestagswahl 2013. Eine Reform der Reform der Reform ist unvermeidlich» [La nueva ley electoral para la elección del Bundestag de 2013. Una reforma de la reforma de la reforma es imprescindible], en: Zeitschrift für Parlamentsfragen 44, 2013.

50 Grotz, F., «Happy End oder endloses Drama? Die Reform des Bundestagswahlsystems» [Happy end o drama sin fin. La reforma del sistema electoral del Bundestag], en: Jesse, E. /STURM, R. (Eds.): Bundestagswahl 2013. Voraussetzungen, Ergebnisse, Folgen [La elección al Bundestag de 2013. Condiciones preliminares, resultados, consecuencias], Nomos, Baden-Baden, 2014. 
